\title{
Best practices on personalization and adaptive interaction techniques in the scope of Smart Homes and Active Assisted Living
}

\author{
Nikolaos Liappas*, José Gabriel Teriús-Padrón*, Eduardo Machado ${ }^{\dagger}$, Mohammad Reza Loghmani ${ }^{\ddagger}$, \\ Rebeca Isabel García-Betances*, Markus Vincze ${ }^{\ddagger}$, Iván Carrillo Quero ${ }^{\dagger}$ and María Fernanda Cabrera-Umpiérrez* \\ *Life Supporting Technologies, ETSI Telecomunicaciones \\ Universidad Politécnica de Madrid, Av. Complutense 30, 28040 Madrid, Spain \\ Email: \{nliappas, jterius, rgarcia, chiqui\} @1st.tfo.upm.es \\ ${ }^{\dagger}$ Department of Innovation and Technology, Ingenieria y Soluciones Informatica, Sevilla, Spain \\ Email: \{eduardo.machado, ivan.carrillo\}@isoin.es \\ $\ddagger V 4 R$, ACIN, Faculty of Electrical Engineering Technische Universität Wien, Vienna, Austria \\ Email: \{loghmani, vincze $\} @$ acin.tuwien.ac.at
}

\begin{abstract}
Assistive systems and emerging technologies are capable of supporting individuals with specific needs and diseases effectively. Nonetheless, in the domain of Smart Homes (SH), the interactions tend to become more complex and difficult to adapt to the users, creating a bottleneck for the efficient use and the acceptability of the interventions. This paper presents best practices on personalization and adaptive interaction techniques in order to homogenize different solutions within the $\mathrm{SH}$, ease the interaction acceptability by adaptation to specific user needs and implement better healthcare interventions able to improve the Quality of Life (QoL). The recommendations arise as a result of previous research studies conducted within the MSCA-ITN project ACROSSING.
\end{abstract}

\section{INTRODUCTION}

Research has been undertaken on multi-modal interaction to enable natural, intuitive, personalized and adaptive interactions to support individuals with specific needs [1] [2] [3]. Notwithstanding, there is a gap between these generic methods, the effective solutions for heterogeneous applications and the different groups of end users. The development of Information and Communication Technology (ICT) interventions in the healthcare domain usually involves and requires all the stakeholders such as designers, developers, caregivers, patients, family and other professionals. Ordinarily, this process applies the User-Centred Design (UCD) methodology [4] by involving the final user as the foundation of the research and development process. Table I summarizes some specific applications that could be adapted and implemented in SHs with respect to the needs of different individuals, related to solutions for people with cognitive impairments, self-management of chronic diseases and assisted living applications.

The potential risk of developing Alzheimer Disease (AD) is high especially for elderly [5], and cognitive impairments are considered to be a precursor to AD. Research on nonpharmacological interventions has pulled in significant consideration as a safe, generally modest and versatile interventions that can support the deficits caused by AD such as daily activ- ities and cognitive decline. Managing the QoL of individuals with cognitive impairments is challenging and risky, especially for the family related caregivers. Elderly people namely with Mild Cognitive Impaired (MCI) have been excluded from accessing the digital technologies because user interfaces do not meet the necessary requirements. Deficits in cognition and functions such as working memory, controlled attention and speed of processing increase the difficulty of elderly to learn new concepts, recall complex operational procedures or navigate complex menu structures. $\mathrm{SH}$, emerging and interacting technologies [6] jointly with assisted living can enhance the QoL and broaden this chronic disease cycle.

Worldwide, over 40 million deaths are caused by chronic diseases per year, where cardiovascular, cancer and respiratory diseases are the three main causes of those deaths [7]. In addition, the WHO indicates that $72 \%$ outdoor air pollutionrelated premature deaths are related to those chronic conditions [8]. Despite the fact that chronic diseases have no cure [7], existing literature demonstrates that individuals with chronic conditions enrolled in Chronic Diseases Self- Management Programs (CDSMP) can improve their health status, health behaviour and reduce the occurrence of relapses and visits to the hospital [9]. Innovative technologies like Internet of Things (IoT), mobile and wearable technologies can provide reliable and effective healthcare services, whilst they can reduce the public healthcare costs [9] [10] [11]. SH ICT and AAL solutions currently offer support and provide an added value in the self-management of chronic diseases improving significantly the QoL of people with these conditions.

The following sections present best practices and intervention examples aimed as advice for personalized and adaptive interactions based on the experiences learned on different research topics related to: intervention systems targeted for people with MCI (section 2), self-management intervention system for Chronic Diseases (section 3), AAL solutions for improving well-being and QoL using robot gaming strategy 
TABLE I

TECHNOLOGICAL APPLICATIONS FOR SMART HOMES TO SUPPORT THE NEEDS OF PEOPLE WITH COGNITIVE IMPAIRMENTS AND CHRONIC DISEASES

\begin{tabular}{ll}
\hline Activity recognition & $\begin{array}{l}\text { Activities such as cooking, and bathing are classified } \\
\text { to be used for prediction purposes and dynamically } \\
\text { proposed interventions within the SH. }\end{array}$ \\
\hline Sleep monitoring & $\begin{array}{l}\text { Monitoring the sleep of the user yields predicting } \\
\text { interventions and can detect abnormal } \\
\text { sleeping patterns. }\end{array}$ \\
\hline Wearable sensing & $\begin{array}{l}\text { Wearing wristbands, carrying smartphones or other } \\
\text { wearables can monitor physical activity and } \\
\text { provide location properties within the house. }\end{array}$ \\
\hline Environmental sensing & $\begin{array}{l}\text { The continuous monitoring of the environmental } \\
\text { parameters offers interventions able to impact the } \\
\text { well-being, comfort and the productivity of the users. }\end{array}$ \\
\hline Security & $\begin{array}{l}\text { Securing the whole system provides a safety feeling } \\
\text { to the individual. }\end{array}$ \\
\hline Physical activity promotion & $\begin{array}{l}\text { The smart home provides physical activities to the } \\
\text { individuals through computerized and monitored } \\
\text { exercises or other instruments like bicycles. }\end{array}$ \\
\hline
\end{tabular}

(section 4). Finally, section 5 delivers a summary of our generic recommendations.

\section{ENHANCING AND SUPPORTING INTERACTIONS FOR PEOPLE WITH COGNITIVE IMPAIRMENTS}

Individuals with cognitive impairments face problems ranging from memory issues to functional difficulties performing daily activities. These problems generate a higher dependence to the daily care, in some cases within a care centre. Shortterm or long-term memory loss, attention deficiencies, lack of adequate information of the disease and its treatments, lack of technology familiarity and adoption, social isolation and lack of physical activity are some issues that people with MCI have to encounter [12]. Transforming these issues to needs, offers researchers the possibility to view a broader aspect of this domain and provide useful information to develop adequate solutions according to their needs.

Commonly, people affected with MCI are elderly that in parallel have to deal with other conditions or diseases. In order to develop an assistive technology targeting elderly people with MCI we must firstly understand the complexity of the ageing process. Ageing is associated with various changes in cognitive abilities, which significantly reduce their ability to understand and navigate under common user interfaces designed over one size fits all approach. Moreover, ageing will likely cause sensory and motor issues that negatively affects individuals' ability to interact with user interfaces based on tactile interaction modalities. In that sense, we must consider not only one, but all user needs related to all the specific conditions of a user, in order to offer a fully accessible and personalized solution.

Designing and implementing technologies for people with cognitive impairments can have different approaches: using or adapting existing solutions and frameworks of previous studies and projects, or supporting seniors with current technology, both mechanical and electronic equipment, within their living environments. In the course of our investigation, interviews, focus groups and technical tests with people with cognitive impairments specifically $\mathrm{MCI}$, revealed us that some devices are more applicable than others regarding the interactions [12]. Acknowledging the previous user specifications and needs, we identified the most suited current technologies and ICT devices to enhance and support user interaction of people with cognitive impairments and are the following:

- Computer or mobile devices (tablet or smartphone): to interact with tablet gestures and virtual keyboards as they are suitable/acceptable for managing the interventions and interacting with different elements (e.g. SH devices) or applications. Smartphones are appropriate when the person is more familiar with mobile phones. Tablets offer the same benefits of mobile phones in terms of easiness of the interaction with touch screens, but they have bigger screens that could be better for these users. This choice it is always the easier to adopt and implement as the cost is low. Computers and laptops with big screens and usual keyboards interaction are other options but only if the users are familiar with computers, then the interaction with them should be straightforward and easy [12].

- Sensors and actuators: this kind of technology can be used to collect information and measurements, perform activity recognition and automatize and control smart environments. The potential of sensors and actuators are their unobtrusiveness and natural interaction that is less demanding in term of cognitive workload. They could act automatically without the intervention of the users. This represents an important characteristic to consider when developing ICT solutions for people with cognitive impairments, since it notably decreases the complexity of user interaction.

- Wearable devices: wristbands, smartwatches, etc. to track the location of the user either indoors or outdoors and provide data to caregivers and family members. They can also track sleeping disturbances which are known to usually lead to memory loss or the nighttime wandering of the individuals. Wearable devices are easy to use devices that can support the interaction with applications or mobile solutions. Furthermore, they are non-invasive and could be easily integrated in users daily activities. In some cases, they also have the capability of unobtrusively collect information of different types such as: biological signals (i.e. heart rate), environmental measurements (i.e. user location, temperature, etc.), physical activity (i.e. number of steps per day), etc.

- Virtual Reality (VR) solutions and environments: are advantageous to deliver cognitive and physical activity therapies through semi-immersive or immersive environments with numerous benefits such as: the identification of objective ecological factors, real time feedback, high interaction with different scenarios while being safe in an personalized environment. Interventions based on VR are well accepted and promoted specifically for cognitive training interventions as they show promising results as opposed to classical papers tasks in cognitive training 
[13].

- Augmented Reality (AR): has been shown promising results as an assistive technology in many different application domains. However, in order to tackle all user needs regarding our target group we should focus on two main assumptions: reduce the solution intrusiveness and design it to be task free, independent of the use case scenario. In this sense, AR solutions together with other kind of technologies are a suitable option to enhance and support user interaction.

\section{A. Implementation examples}

Smart Home-based Virtual Assistant for people with MCI: The solution consists of a virtual assistant running in a modern cross-platform way within a SH environment. It is deployed around the SH and it is connected with it through sensors (i.e infared or ultrasonic sensors capable to detect the presence or motion within range, pressure sensors distributed in the house and can detect steps or smoke sensors to alarm the users etc.) using a middle-ware. The interface with the user is displayed all over the house, including PCs and TVs.

The assistant requires an input: the clinic history of the user and the cognitive functions affected. This will be used to adapt the assistant better to the needs: for example if the user has greater decline in memory rather than spatial disorientation, then the interventions will be adapted and promoted to train more with cognitive and physical exercises while reminders and alarms will be in a higher priority. During a normal day of an MCI individual living with the assistant: the house is sensing the user through various sensors and communicates with the back-end of the assistant with a middle-ware solution. The assistant will personalize the interventions in real time and propose interventions accordingly. The user will have to perform the activities suggested by the assistant (i.e. to go for a $2 \mathrm{~km}$ walk outdoors or in a park) and the assistant will follow up if the activity was completed or not in order to continue adapting the intervention. If the activity is not completed then a caregiver or a notification to a family member will be sent. Smart voice assistants such as Google Assistant can be embedded at this functionality level to give a boost in the communication part or in the reminders' routines. Lastly, to adapt the personalized virtual assistant in other conditions and diseases, the following steps are recommended: a) determine with condition experts the goal of the assistant; b) identify mobility restrictions c) identify light sensitivity requirements; d) determine duration of use;

Augmented Reality and Head worn eye tracker to assist elderly people in daily activities: In this illustration, we present a solution based on AR and a head worn eye tracker as a means to assist elderly people in their daily activities. We further include a multi-modal interaction approach to address the difficulties caused by sensory and motor decline. The components and the workflow are presented in Figure 1.

It is important to mention that our solution is designed to target task free and ongoing conditions. In that sense, it is crucial to combine technologies that can extract the maximum

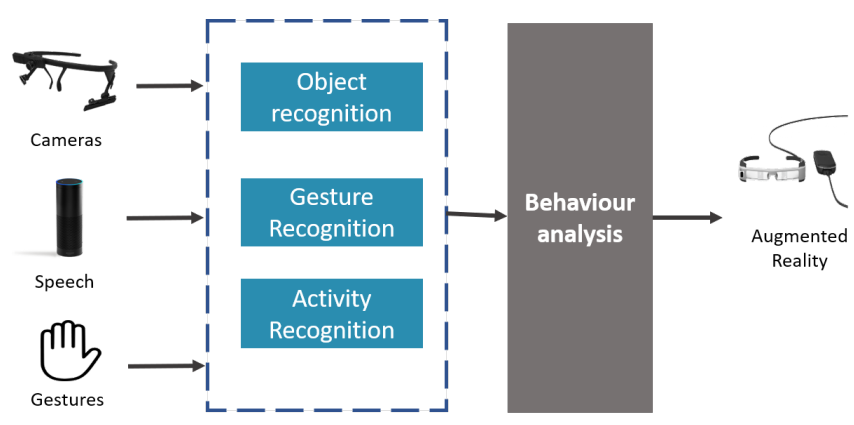

Fig. 1. System architecture based on multi-modal approach. The flow is represented from the input data eye tracker cameras pushing it to different modules. The output exposes different interaction modalities such as augmented reality (visual modality)

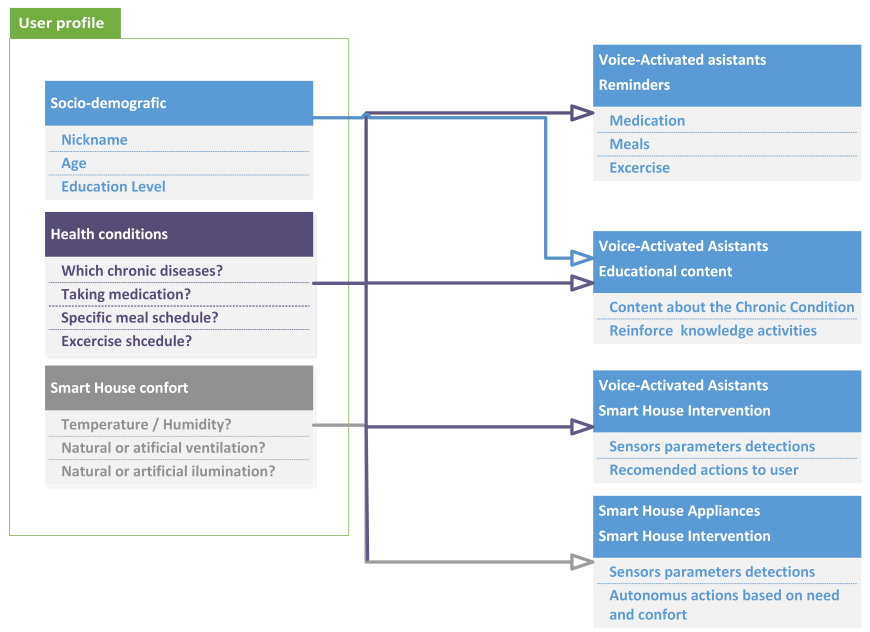

Fig. 2. Road-map for personalized intervention using a voice activated assistant

information about the current user context and his intentions without being intrusive. Following this premise, we use a head worn and eye tracker cameras to capture information about user visual attention and his immediate surroundings. As follows, the proposed solution is able to infer user behaviour as well as his context. As user interface, AR glasses is proposed due to its advantages in terms of information visualisation and user engagement.

The following is an example of the workflow that can be applied in daily activities such as cooking. This example used as a paradigm in the workflow presented in Figure 1.

- The AR glasses present information to the user regarding the execution of a specific step of a recipe in different formats such as images and text.

- In case of a positive response by the user, the system proceeds and displays relevant information.

- In case of a negative response, the system identifies the source of the failure (i.e. missed an ingredient, forgot to turn on the oven, wrong measurement of water etc.) and alerts the user.

The purpose of this solution may change based on the environment and on users needs. The combined technologies 
make this solution easy to be personalized to assist the user in different domain applications. Other major advantage comparing to popular assistive technologies, is that computers and tablets need constant interaction and manipulation, which can make them unsuitable as medium of content delivery for the elderly. In the opposite direction, AR together with the gesture and speech modality provide an easy and intuitive interface reducing elderly resistance of technology acceptance.

\section{PERSONALIZED SELF-MANAGEMENT AND INTERVENTION FOR INDIVIDUALS WITH CHRONIC DISEASES}

The self-management of chronic diseases is one of the most used techniques to improve the QoL of individuals suffering of this type of diseases. Existing solutions focus on the empowerment of the user by providing the necessary tools to effectively control the illness by reducing the number of relapses of the disease and hospital admissions as well [14]. Self-management skills include problem solving, decision making, resource utilization and taking actions. Furthermore, it can be applied in various aspects to improve users conditions, such as, drugs, symptoms and lifestyle [15].

During the process of the development of ICT solutions for the self-management of chronic diseases, it is important to take into consideration environmental factors such as air quality, temperature, humidity, pollution levels, among others. They are considered with greater emphasis due to the increasingly close relationship between these factors and the health of the individuals with chronic diseases such as heart failure, respiratory failure and others [16]. The main objective of chronic self-management techniques is to empower users to manage their condition in the best possible way. To accomplish this goal within a $\mathrm{SH}$ scenario, the technology requirements are the following:

- Voice activated assistant: for the user interaction with the system. Technologies such as Google Assistant [17] can be used in both mobile phones and Google Home devices, offering the possibility to interact with the device of their preference.

- Sensor technology: air quality, temperature, humidity and motion sensors that will provide relevant information to manage the environmental conditions.

- Actuators: to control doors, windows and other home appliances in order to respond to the user or system commands.

In the following subsection we present the recommendations for implementing a personalized system for intervention using voice activated assistant within a Smart Home.

\section{A. Recommendations and Implementation example}

Voice-activated assistant to support self-management of chronic diseases: This example consists of a system that helps the self-management of individuals with chronic diseases, based on the measurement of air quality parameters within the $\mathrm{SH}$. This system is composed of a voice-activated assistant, air quality sensors and actuators to control household appliances.
The voice-activated assistant will serve as a tool for the interaction by which interventions such as reminders for medication, food, educational content and warnings will be delivered to users. On the other hand, the SH can autonomously maintain an optimal environmental condition (e.g. temperature, humidity, air quality) considering the users preferences and needs based on the specific health condition. Figure 2 demonstrates a personalized system that can be implemented including different interaction or interventions that can be done through a voice activated assistant device in a $\mathrm{SH}$.

Specifically, the solution is based on four principal elements, described as follow:

1) User classification and profile: personalization is essential since each disease requires different actions in terms of treatment and management. Therefore, it is important to classify each user based on the medical profile and medical treatment. This profile should be precise in order to easily classify users based on diseases' specific conditions.

2) Educational content: the system should be able to give educational content to empower the user to control the illness. This content is based on the profile classification, since each chronic disease is different.

3) Reminders: The system should be able to remind medication intake, exercise performance, medical appointments, among others, depending on the condition of the user.

4) $\mathrm{SH}$ intervention: The $\mathrm{SH}$ should be able to manage the balance between medical recommendations and user comfort in terms of environment using different kind of sensors and actuators that will control smart devices. Measurements such as temperature, humidity, pollution levels, etc. should be constantly collected and adjusted based on user preferences and medical recommendations. On the other hand, it is recommended to manage the $\mathrm{SH}$ intervention in 2 levels:

a) Level 1, recommendation: the $\mathrm{SH}$ through the voice activated assistant provides the user a recommended action based on sensors' measurements and waits for the user input on how to proceed further.

b) Level 2, autonomous action: if the sensors detect an unhealthy scenario and the user does not act on Level 1, the SH automatically will perform the actions to stabilize the environment.

\section{ROBOT GAMING STRATEGIES TO ENHANCE ELDERLY'S WELL-BEING AND QUALITY OF LIFE}

In order to guarantee the QoL of elderly people, it is important to consider not only the cognitive decline, but also the physical decline of the user. This can be either due to the natural process of ageing or due to specific disabilities.

The use of an active agent, such as a mobile robot, is a natural choice to support elderly people to physically act in the home environment. To this purpose, the robot should be 
TABLE II

SUMMARY OF GUIDELINES

Generic guidelines for specific use cases

\begin{tabular}{|c|c|}
\hline \multicolumn{2}{|r|}{ Generic guidelines for specific use cases } \\
\hline Use case & Recommendation \\
\hline 1. Cognitive decline & $\begin{array}{l}\text { (R1.1) - Implement cognitive training exercises with interfaces of tablet, mobile phones, computers and VR. Touch gestures are acceptable and } \\
\text { recommended. (R1.2) - VR is the most promising solution because of the encouraging results while the classic paper tasks should be avoided. } \\
\text { (R2.1) - Implement mechanisms to monitor indoors activity all over the home. (R2.2) - Provide interactive help to assist the users accomplish the daily }\end{array}$ \\
\hline 2. Inabilities with daily activities & $\begin{array}{l}\text { activities, this can be achieved with a virtual assistant running in the smart house. To achieve that, specific modelling and training is } \\
\text { required when performing daily activities. (R2.3) - Emerging mechanisms should notify the caregivers or the family in case of emergency } \\
\text { such as fire when cooking. }\end{array}$ \\
\hline 3. Assistance with daily activities & $\begin{array}{l}\text { (R3.1) - Use Augmented reality glasses for assisting and monitoring the user in daily live activities. Augmented reality allows for side-by-side } \\
\text { training and execution by layering additional information on top of a user's direct view. (R3.2) - Implement multi modal interaction techniques } \\
\text { with Augmented Reality glasses (i.e. gestures and voice). This will improve the user experience as well as his engagement. }\end{array}$ \\
\hline 4. Chronic Diseases self-management & $\begin{array}{l}\text { (R4.1) - The system should be able to define a user profile based on the chronic condition. (R4.2) - Use voice-activated assistant for reminders, educational } \\
\text { content and raise awareness, warnings and alerts. The SH should be able to balances between recommended ambient conditions and user } \\
\text { preference. (R4.3) - SH autonomous interventions should be manage in two level: Level 1: recommendation to the user (using voice assistant) and } \\
\text { make intervention if it is authorized. Lever 2: autonomous action based on sensor measurements, inform to user about the action done. }\end{array}$ \\
\hline 5. Interfacing with an active agent & $\begin{array}{l}\text { (R5.1) - To maximise the ease of interaction with an active agent, such as a service robot, use a combination of speech and visual information on a } \\
\text { tablet. (R5.2) - Interaction via speech is natural for the user, while the tablet can be used to make the communication effective both by providing a } \\
\text { means for the robot to give visual feedback and for the user to easily provide more precise input. }\end{array}$ \\
\hline
\end{tabular}

able to understand the environment and act on it. However, the Human-Robot Interaction (HRI) part should not be neglected: the robot needs not only to be able to complete a given task, but the user should also be able to use it without the need for a specific training. The technological requirements to fulfil are:

- Mobile robotic platform.

- RGB-D sensor(s): main source of information able to provide RGB and depth streams that are used from the robot to understand the surrounding environment and reason on it.

- Robotic manipulator attached to the platform: it could be useful for grasping, but also for a second in-board camera that can be easily moved around to further explore the environment even if the platform is stationary.

- Speakers: one of the channels of communication that the robot can use to provide information to the user.

- Microphones: one of the channels of communication that the robot can use to receive information from the user.

- Tablet: a means for the robot both to provide information and to receive input for the user; All the above-mentioned devices and sensors are part of the robots equipment and should be thought as belonging to the same platform.

The design of the interaction protocol and the specific modes should be based on the literature on human-computer interaction for older adults. While speech is a natural interaction mode, a physical input device, such as a touchscreen, is proven to be more effective to take precise inputs from the user [18] [19]. In order to increase the acceptance of the user, prerecorded human voice should be preferred to synthetic speech generation for the robots speech output [20]. Finally, the visual information provided by a tablet allows more complete and robust communication.

\section{A. Implementation example}

The effectiveness of the interaction between the robot and the user is of great relevance for positively affecting the users well-being [21]. Let us consider the object search task as a mean to design a human-robot interaction protocol [22]. A high-level scheme of the object search task is shown in Figure 3 .

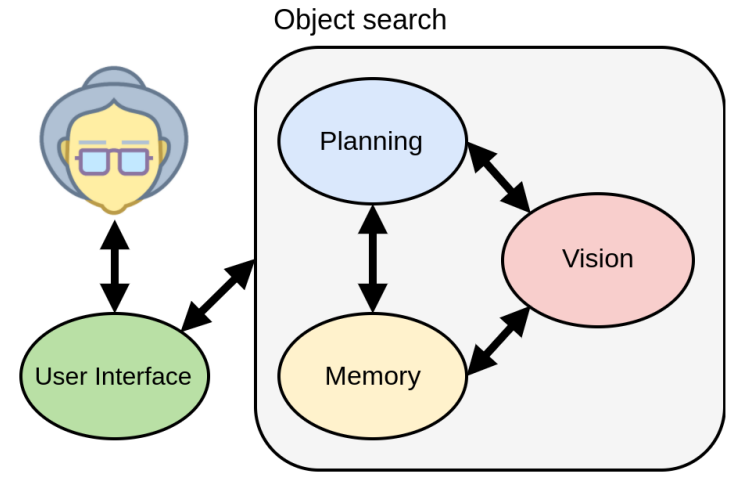

Fig. 3. High-level scheme exposing the different modules involved in the object search task. Modules are represented by ellipses while double arrows indicate a bidirectional flow of information between connected entities. Image taken from [22]

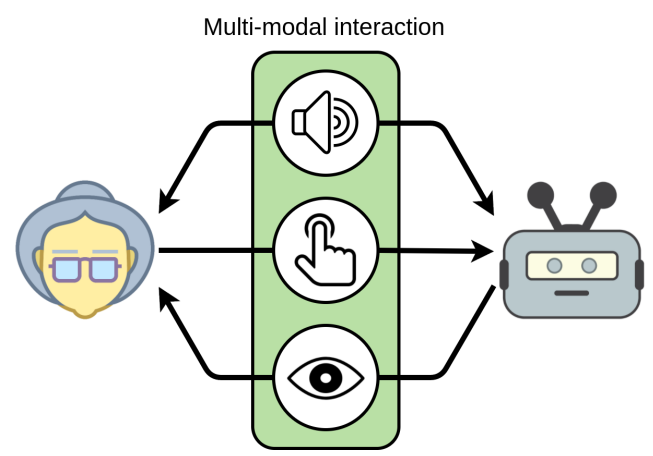

Fig. 4. Scheme representing the multi-modal interaction between the user and the robot. The robot outputs complementary information through two distinct modes, audio (speech) and visual (tablet), while the users input is taken through the touch mode (tablet). Image taken from [22]

Although this task is used to explain the user interface with the robot, the principle discussed in this section is general and can be applied to any task in the context of service robots for elderly. For the robot to assist the user in searching for a lost object, two distinct interaction phases are needed: object selection and location report. In the object selection phase, the robot acquires the information about which object the user is 
interested in using the following steps:

1) the user says the name of the object and a speech recognition API, e.g. Google Cloud Speech, is used to process the information.

2) Speech and display of images on a tablet are used by the robot to disambiguate between the possible objects that match the speech recognition results.

3) The identity of the object is confirmed by the user using the tactile input of a tablet.

In the location report phase, the robot reports back the location of the object to the user after completing the search using the following steps:

1) in case of positive outcome, the robot reports to the user the name of the room in which the object is located, e.g. kitchen. In addition, this information is complemented by showing pictures of the object in its location on the tablet;

2) in case of negative outcome, the robot simply reports that the object was not found through speech by using the incorporated speakers.

It is worth noticing that the whole interaction is based on a multi-modal interface that involves audio, visual and tactile signals, as represented in Figure 4.

\section{SUMMARY AND CONCLUSION}

Best practices for the development of ICT solutions for individuals with cognitive impairments, self-management of chronic diseases, and AAL applications are presented together with implementation examples based on ongoing research within the MSCA-ITN ACROSSING project. Our research provides recommendations to develop ICT solutions which will provide better care for the ageing population, specifically addressing the needs of each conditions. Table II presents a summary of the best practices and recommendations explained in the previous sections. The recommendations are presented per use case with the relevant elements to consider based on the specific target group.

\section{ACKNOWLEDGMENT}

This work is funded by the European Union Horizon 2020 research and innovation program under the Marie SkłodowskaCurie grant agreement ACROSSING No 676157.

\section{REFERENCES}

[1] D. Calvaresi, D. Cesarini, P. Sernani, M. Marinoni, A. F. Dragoni, and A. Sturm, "Exploring the ambient assisted living domain: a systematic review," Journal of Ambient Intelligence and Humanized Computing, vol. 8, no. 2, pp. 239-257, 2017.

[2] N. Carbonell, "Ambient multimodality: towards advancing computer accessibility and assisted living," Universal Access in the Information Society, vol. 5, no. 1, pp. 96-104, 2006.

[3] M. Blumendorf and S. Albayrak, "Towards a framework for the development of adaptive multimodal user interfaces for ambient assisted living environments," in International Conference on Universal Access in Human-Computer Interaction, pp. 150-159, Springer, 2009.

[4] D. A. Norman and S. W. Draper, User centered system design: New perspectives on human-computer interaction. CRC Press, 1986.
[5] M. J. Katz, R. B. Lipton, C. B. Hall, M. E. Zimmerman, A. E. Sanders, J. Verghese, D. W. Dickson, and C. A. Derby, "Age and sex specific prevalence and incidence of mild cognitive impairment, dementia and alzheimers dementia in blacks and whites: A report from the einstein aging study," Alzheimer disease and associated disorders, vol. 26, no. 4, p. $335,2012$.

[6] M. Benoit, R. Guerchouche, P.-D. Petit, E. Chapoulie, V. Manera, G. Chaurasia, G. Drettakis, and P. Robert, "Is it possible to use highly realistic virtual reality in the elderly? a feasibility study with imagebased rendering," Neuropsychiatric disease and treatment, vol. 11, p. 557, 2015.

[7] W. H. Organization, "Noncommunicable diseases. world health organization," 2017. [Online; accessed 01-April-2019].

[8] W. H. Organization, "Ambient air pollution: a global assessment of exposure and burden of disease. world health organization," 2016. [Online; accessed 01-April-2019].

[9] M. M. Baig, H. GholamHosseini, A. A. Moqeem, F. Mirza, and M. Lindén, "A systematic review of wearable patient monitoring systems-current challenges and opportunities for clinical adoption," Journal of medical systems, vol. 41, no. 7, p. 115, 2017.

[10] Y. Yuehong, Y. Zeng, X. Chen, and Y. Fan, "The internet of things in healthcare: An overview," Journal of Industrial Information Integration, vol. 1, pp. 3-13, 2016.

[11] J. Wang, Y. Wang, C. Wei, N. Yao, A. Yuan, Y. Shan, and C. Yuan, "Smartphone interventions for long-term health management of chronic diseases: an integrative review," Telemedicine and e-Health, vol. 20, no. 6 , pp. 570-583, 2014.

[12] N. Liappas, R. I. García-Betances, J. G. Teriús-Padrón, and M. F. Cabrera-Umpiérrez, "Studying the technological barriers and needs of people with dementia: A quantitative study," in 2018 IEEE International Conference on Pervasive Computing and Communications Workshops (PerCom Workshops), pp. 884-889, IEEE, 2018.

[13] R. I. García-Betances, M. T. Arredondo Waldmeyer, G. Fico, and M. F. Cabrera-Umpiérrez, "A succinct overview of virtual reality technology use in alzheimers disease," Frontiers in aging neuroscience, vol. 7, p. 80, 2015.

[14] P. A. Grady and L. L. Gough, "Self-management: a comprehensive approach to management of chronic conditions," American Journal of Public Health, vol. 104, no. 8, pp. e25-e31, 2014.

[15] J. G. Teriús-Padrón, G. Kapidis, S. Fallmann, E. Merdivan, S. Hanke, R. I. García-Betances, and M. F. Cabrera-Umpiérrez, "Towards selfmanagement of chronic diseases in smart homes: Physical exercise monitoring for chronic obstruction pulmonary disease patients," in 2018 IEEE International Conference on Pervasive Computing and Communications Workshops (PerCom Workshops), pp. 776-781, IEEE, 2018.

[16] Z. Zheng, X. Zhang, J. Wang, A. Dandekar, H. Kim, Y. Qiu, X. Xu, Y. Cui, A. Wang, L. C. Chen, et al., "Exposure to fine airborne particulate matters induces hepatic fibrosis in murine models," Journal of hepatology, vol. 63, no. 6, pp. 1397-1404, 2015.

[17] "Google assistant." [Online; accessed 01-April-2019].

[18] H. Huttenrauch and K. S. Eklundh, "Fetch-and-carry with cero: Observations from a long-term user study with a service robot," in Proceedings. 11th IEEE International Workshop on Robot and Human Interactive Communication, pp. 158-163, IEEE, 2002.

[19] M. Montemerlo, J. Pineau, N. Roy, S. Thrun, and V. Verma, "Experiences with a mobile robotic guide for the elderly," in Eighteenth National Conference on Artificial Intelligence, (Menlo Park, CA, USA), pp. 587592, American Association for Artificial Intelligence, 2002.

[20] J. Eriksson, M. J. Mataric, and C. J. Winstein, "Hands-off assistive robotics for post-stroke arm rehabilitation," in 9th International Conference on Rehabilitation Robotics, 2005. ICORR 2005., pp. 21-24, IEEE, 2005.

[21] D. Feil-Seifer and M. J. Mataric, "Defining socially assistive robotics," in 9th International Conference on Rehabilitation Robotics, 2005. ICORR 2005., pp. 465-468, IEEE, 2005.

[22] M. R. Loghmani, T. Patten, and M. Vincze, "Towards socially assistive robots for elderly: An end-to-end object search framework," in 2018 IEEE International Conference on Pervasive Computing and Communications Workshops (PerCom Workshops), pp. 890-895, IEEE, 2018. 